\title{
Greedy Algorithm for Local Contrast Enhancement of Images
}

\author{
Kartic Subr, Aditi Majumder, and Sandy Irani \\ School of Information and Computer Science, University of California, Irvine
}

\begin{abstract}
We present a technique that achieves local contrast enhancement by representing it as an optimization problem. For this, we first introduce a scalar objective function that estimates the average local contrast of the image; to achieve the contrast enhancement, we seek to maximize this objective function subject to strict constraints on the local gradients and the color range of the image. The former constraint controls the amount of contrast enhancement achieved while the latter prevents over or under saturation of the colors as a result of the enhancement. We propose a greedy iterative algorithm, controlled by a single parameter, to solve this optimization problem. Thus, our contrast enhancement is achieved without explicitly segmenting the image either in the spatial (multi-scale) or frequency (multi-resolution) domain. We demonstrate our method on both gray and color images and compare it with other existing global and local contrast enhancement techniques.
\end{abstract}

\section{Introduction}

The problem of contrast enhancement of images enjoys much attention; its applications span a wide gamut, ranging from improving visual quality of photographs acquired with poor illumination [1] to medical imaging [2].

Common techniques for global contrast enhancements like global stretching and histogram equalization do not always produce good results, especially for images with large spatial variation in contrast. A number of local contrast enhancement methods have been proposed to address exactly this issue. Most of them explicitly perform image segmentation either in the spatial(multi-scale) or frequency(multi-resolution) domain followed by a contrast enhancement operation on each segment. The approaches differ mainly in the way they choose to generate the multi-scale or multi-resolution image representation (anisotropic diffusion [2], non-linear pyramidal techniques 3 , multi-scale morphological techniques [45], multi-resolution splines [6], mountain clustering [7], retinex theory 89 ) or in the way they enhance contrast after segmentation (morphological operators [5], wavelet transformations [10], curvelet transformations [11, k-sigma clipping [89, fuzzy logic [127, genetic algorithms [13]).

In this paper we present a local contrast enhancement method driven by a scalar objective function that estimates the local average contrast of an image. Our goal is to enhance the local gradients, which are directly related to the local contrast of an image. Methods that manipulate the local gradients 14 15. need 
to integrate the manipulated gradient field to construct the enhanced image. This is an approximately invertible problem that requires solving the poisson equation dealing with differential equations of potentially millions of variables.

Instead, we achieve gradient enhancement by trying to maximize a simple objective function. The main contributions of this paper are:

- a simple, scalar objective function to estimate and evaluate the average local contrast of an images,

- an efficient greedy algorithm to enhance contrast by seeking to maximize the above objective function.

We present our contrast enhancement algorithm for gray image in Section 2. In Section 3 the extension of this method to color images is described, followed by the results in Section 4 Finally, we conclude with future work in Section 5 .

\section{Contrast Enhancement of Gray Images}

\subsection{The Optimization Problem}

The perception of contrast is directly related to the local gradient of an image [16. Our objective is to enhance the local gradients of an image subject to strict constraints that prevent both over/under-saturation and unbounded enhancement of the gray values. Thus, we propose to maximize the objective function

$$
f(\Omega)=\frac{1}{4|\Omega|} \sum_{p \in \Omega} \sum_{q \in N_{4}(p)} \frac{I^{\prime}(p)-I^{\prime}(q)}{I(p)-I(q)}
$$

subject to the constraints,

$$
\begin{gathered}
1 \leq \frac{I^{\prime}(p)-I^{\prime}(q)}{I(p)-I(q)} \leq(1+\delta) \\
L \leq I^{\prime}(p) \leq U
\end{gathered}
$$

where scalar functions $I(p)$ and $I^{\prime}(p)$ represent the gray values at pixel $p$ of the input and output images respectively, $\Omega$ denotes set of pixels that makes up the image, $|\Omega|$ denotes the cardinality of $\Omega, N_{4}(p)$ denotes the set of four neighbors of $p, L$ and $U$ are the lower and upper bounds on the gray values (eg. $L=0$ and $U=255$ for 8 bit gray values between 0 and 255), and $\delta>0$ is the single parameter that controls the amount of enhancement achieved. We seek to maximize our objective function by pronouncing the local gradient around a pixel in the input image to the maximum possible degree. The constraint defined by Equation 2 assures a bounded enhancement of gradients. The lower bound ensures that the signs of the gradients are preserved and that the gradients are never shrunk. The upper bound ensures a bounded enhancement of contrast controlled by the parameter $\delta$. The constraint defined by Equation 3 ensures that the output image does not have saturated intensity values. 

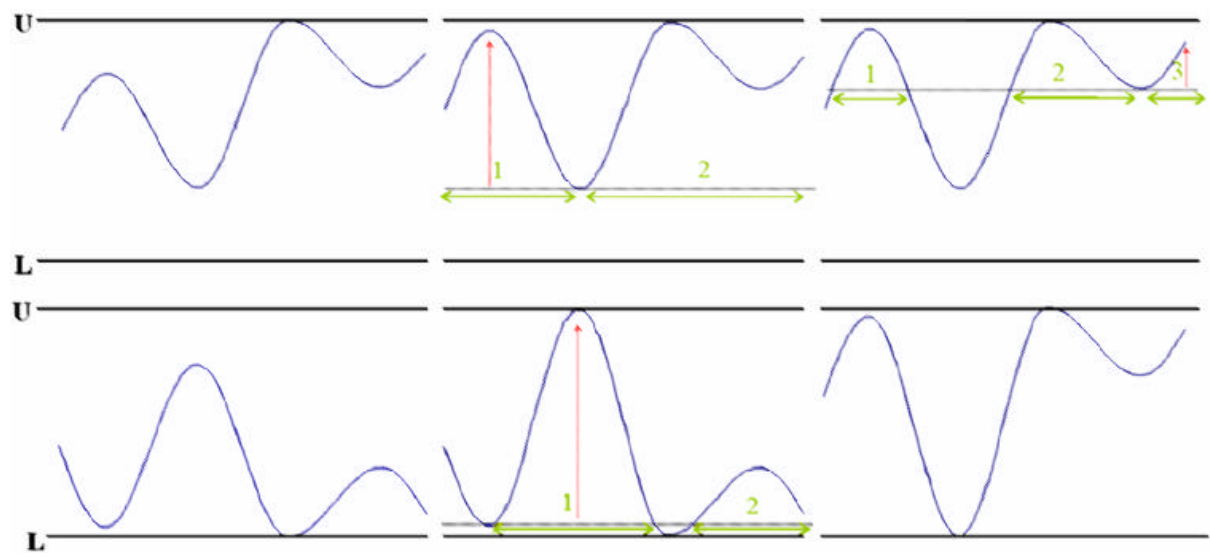

$\mathbf{L}$

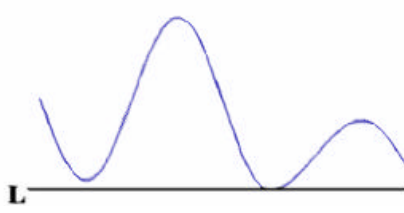

Fig. 1. Graphs showing key steps in our algorithm when applied to a 1D signal. Hillocks formed at each stage are numbered. Their extent is shown with green arrows and enhancement with red arrows. Top Row(left to right): Input signal; sweep plane through first minima. Note that hillock 1 is pushed up as much as possible so that the $\delta$ constraint is not violated while hillock 2 cannot be enhanced at all since it will violate the saturation constraint; sweep plane through second minima. Note that hillocks 1 and 2 cannot be enhanced. However hillock 2 from the previous step has split into 2 and 3 of which the latter can be enhanced. Bottom Row(left to right): Invert Signal from previous step; sweep plane through first minima in the inverted signal; output signal obtained by re-inverting.

\subsection{The Algorithm}

We design a greedy algorithm to solve the optimization problem in 2.1. Our algorithm is based on the fundamental observation that given two neighboring pixels with gray values $r$ and $s, s \neq r$, scaling them both by a factor of $(1+\delta)$ results in $r^{\prime}$ and $s^{\prime}$ such that

$$
\frac{r^{\prime}-s^{\prime}}{r-s}=(1+\delta)
$$

Thus if we simply scale the values $I(p), \forall p \in \Omega$, by a factor of $(1+\delta)$, we obtain the maximum possible value for $f(\Omega)$. However, this could cause violation of Equation 3 at $p$, leading to saturation of intensity at that point. To avoid this, we adopt an iterative strategy, employing a greedy approach at each iteration.

Let us visualize the image $I$ as a height-field sampled at the grid points of a $m \times n$ uniform grid. This set of samples represents $\Omega$ for a $m \times n$ rectangular image. Thus, the height at every pixel $p \in \Omega, I(p)$, is within $L$ and $U$. A one dimensional example of the algorithm is shown in Figure 1.

For each iteration, we consider $b, L \leq b \leq U$. Next, we generate an $m \times n$ matrix $R$ by marking the regions of $I$ which are above the plane $b$ as

$$
R(i, j)=\left\{\begin{array}{l}
1 \text { if } I(i, j)>b \\
0 \text { if } I(i, j) \leq b
\end{array}\right.
$$




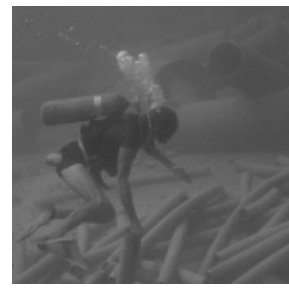

(a)

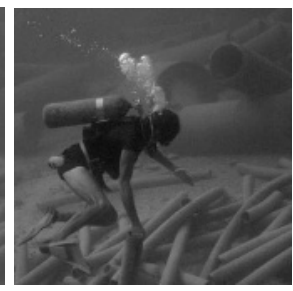

(b)

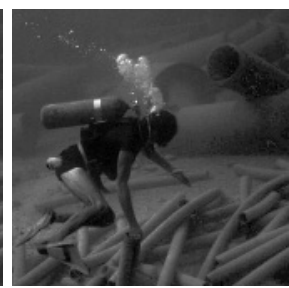

(c)

Fig. 2. The original gray image (a), enhanced gray image using $\delta$ of 0.3 (b) and 2 (c). Note that parts of the image that have achieved saturation for $\delta=0.3$ do not undergo anymore enhancement or show any saturation artifact for higher delta of 2 . Yet, note further enhancement of areas like the steel band on the oxygen cylinder, the driver's thigh and the pipes in the background. The same method applied to the red, green and blue channel of a color image - the original image (d), enhanced image using $\delta$ of 0.4 (e) and 2 (f) - note the differences in the yellow regions of the white flower and the venation on the leaves are further enhanced.

In the example in Figure 1 these are the points above the green line. Next, we find the non-zero connected components in R, and label them uniquely. Let us call each such component, $h_{i}^{b}$, a hillock; $i$ denotes the component number and $b$ denotes the thresholding value used to define the hillocks. In Figure 1, the hillocks are shown numbered. Next, these hillocks are pushed up such that no pixel belonging to the hillock has the gradient around it enhanced by a factor more than $(1+\delta)$ and no pixel is pushed up beyond $U$.

Our method involves iteratively sweeping threshold planes from $L$ through $U$ and greedily scaling the hillocks respecting the constraints at each sweep. Note that as we sweep successive planes, a hillock $h_{i}^{b}$ can split into $h_{j}^{b+1}$ and $h_{k}^{b+1}$ or remain unchanged. But, two hillocks $h_{i}^{b}$ and $h_{j}^{b}$ can never merge to form $h_{k}^{b+1}$. This results from the fact that our threshold value increases from one sweep to the next and hence the pixels examined in an iteration are the subset of the pixels examined in the previous iterations. This observation helps us to perform two important optimizations. First, we obtain the new hillocks by only searching amongst hillocks from the immediately preceding sweep. Second, we store information about how much a hillock has been scaled as one floating point value per hillock. By ensuring that this value never exceeds $(1+\delta)$ we avoid explicitly checking for gradient constraints at each pixel in each iteration. We omit these optimizations in the pseudo-code shown in Figure 3 for simplicity.

For low values of $b$, enhancement achieved on hillocks might not be $(1+\delta)$ because of the increased chances of a peak close to $U$ for large sized hillocks. As $b$ increases, the large connected components are divided so that the smaller hillocks can now be enhanced more than before(see top row of Fig 1). This constitutes the upward sweep in our algorithm which enhances only the local hillocks of $I$ and the image thus generated is denoted by $I_{1}$. 


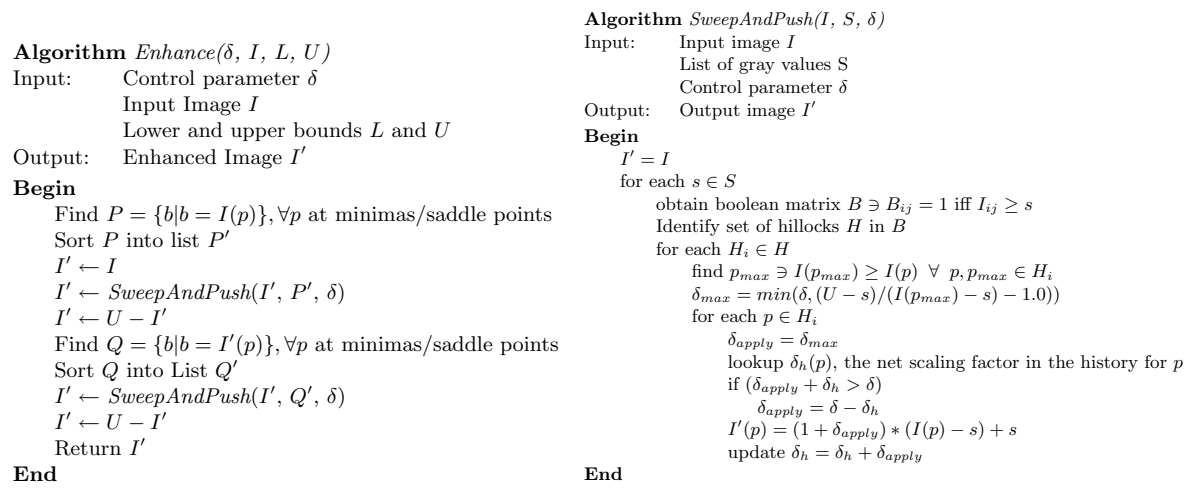

Fig. 3. The Algorithm

Further enhancement can be achieved by enhancing the local valleys also. So, the second stage of the our method applies the same technique to the complement of $I_{1}=U-I_{1}(p)$ to generate the output $I_{2} . I_{2}$ is complemented again to generate the enhanced output image $I^{\prime}=U-I_{2}(p)$ (see bottom row of Fig 1).

We perform $U-L$ sweeps to generate each of $I_{1}$ and $I_{2}$. In each sweep we identify connected components in a $m \times n$ matrix. Thus, the time-complexity of our algorithm is theoretically $O((U-L)(m n+\log (U-L)))$. The logarithmic term arises from the need to sort lists $P$ and $Q$ (see Fig. 3) and is typically dominated by the $m n$ term. However, we observe that hillocks split at local points of minima or saddle points. So, we sweep planes only at specific values of $b$ where some points in the height field attain a local minima or saddle point. This helps us to achieve an improved time complexity of $O(s(m n+\log (s))))$ where $s$ is the number of planes swept (number of local maximas, local minimas and saddle points in the input image). These optimizations are incorporated in the pseudocode of the algorithm in the Figure 3 .

\section{Extension to Color Images}

One obvious way to extend the algorithm presented in Section 2.2 to color images is to apply the method independently to three different color channels. However, doing this does not assure hue preservation and results in hue shift, especially with higher values of $\delta$, as illustrated in Figure 4. To overcome this problem we apply our method to the luminance component of the image only. We first linearly transform the RGB values to CIE XYZ space [17] to obtain the luminance $(Y)$ and the chromaticity coordinate $\left(x=\frac{X}{X+Y+Z}\right.$ and $\left.y=\frac{Y}{X+Y+Z}\right)$, and then apply our method only to $Y$ keeping $x$ and $y$ constant, and finally convert the image back to the RGB space. However, in this case, the constraint in Equation 3 needs to be modified so that the resulting enhanced color lies within the 


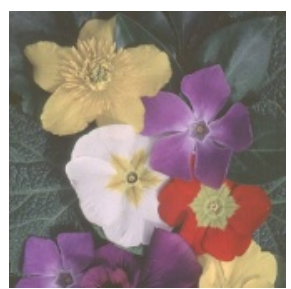

(a)

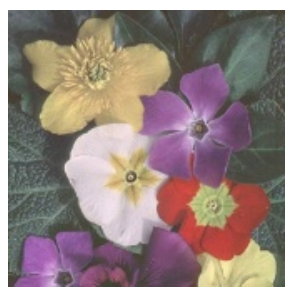

(b)

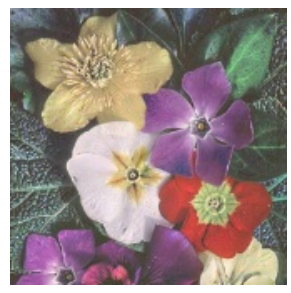

(c)

Fig. 4. Results of applying the algorithm from Section 2.2 on the red, green and blue channels separately. (a) Original image (b) Channels enhanced with a $\delta$ of 0.4 (c) Channels enhanced with a $\delta$ of 2 . Note the artifacts on the petals of the yellow flower and on the leaf venation.

color gamut of the display device. Here we describe the formulation for color images.

The primaries of the display device are defined by three vectors in the XYZ color space $\vec{R}=\left(X_{R}, Y_{R}, Z_{R}\right), \vec{G}=\left(X_{G}, Y_{G}, Z_{G}\right)$ and $\vec{B}=\left(X_{B}, Y_{B}, Z_{B}\right)$. The transformation from RGB to XYZ space is defined by a $3 \times 3$ matrix whose rows correspond to $\vec{R}, \vec{G}$ and $\vec{B}$. Any color in the XYZ space that can be expressed as a convex combination of $\vec{R}, \vec{G}$ and $\vec{B}$ is within the color gamut of the display device. Note that scaling a vector in the XYZ spaces changes its luminance only, keeping the chrominance unchanged. This is achieved by scaling $Y$ while keeping $(x, y)$ of a color constant. In addition, to satisfy the saturation constraint, we assure that the enhanced color lies within the parallelopiped defined by the convex combination of $\vec{R}, \vec{G}$ and $\vec{B}$.

Thus, the color at pixel $p$, given by $C(p)=(X, Y, Z)$ is to be enhanced to $C^{\prime}(p)=\left(X^{\prime}, Y^{\prime}, Z^{\prime}\right)$ by enhancing $Y$ to $Y^{\prime}$ such that the objective function

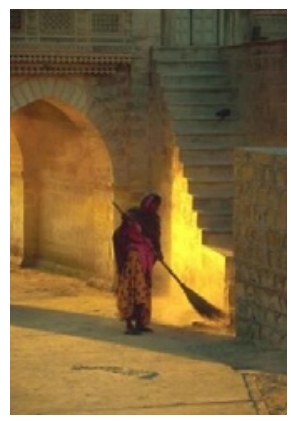

(a)

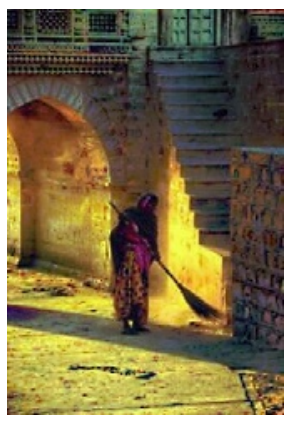

(b)

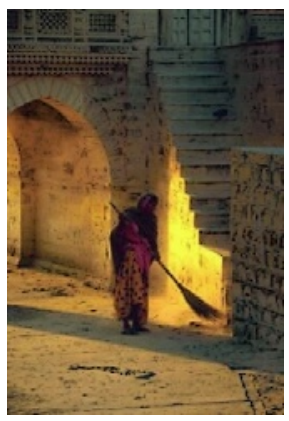

(c)

Fig. 5. (a) Original image, (b) algorithm from Sec. 2.2 applied with $\delta=2$ on the red, green and blue channels separately. Note the significant hue shift towards purple in the stairs, arch and wall and towards green on the ledge above the stairs, (c) Using the method described in Sec 3 with $\delta=2$ (separating the image into luminance and chrominance and applying the method to the former). Note that hue is preserved. 


$$
f(\Omega)=\frac{1}{4|\Omega|} \sum_{p \in \Omega} \sum_{q \in N_{4}(p)} \frac{Y^{\prime}(p)-Y^{\prime}(q)}{Y(p)-Y(q)}
$$

is maximized subject to a perceptual constraint

$$
1 \leq \frac{Y^{\prime}(p)-Y^{\prime}(q)}{Y(p)-Y(q)} \leq(1+\delta)
$$

and a saturation constraint

$$
\left(X^{\prime}, Y^{\prime}, Z^{\prime}\right)=c_{R} \vec{R}+c_{G} \vec{G}+c_{B} \vec{B}, \quad 0.0 \leq c_{R}, c_{G}, c_{B} \leq 1.0
$$

Thus by changing just the saturation constraint, we achieve contrast enhancement of color images without saturation artifacts (Figure 5).

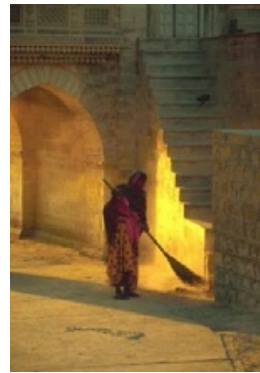

(a)

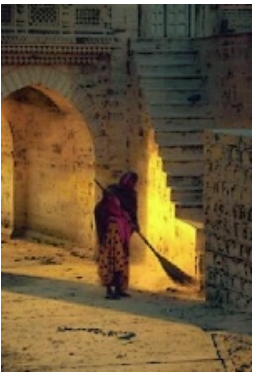

(b)

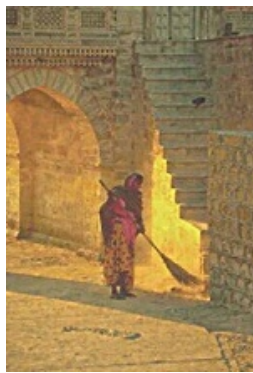

(c)

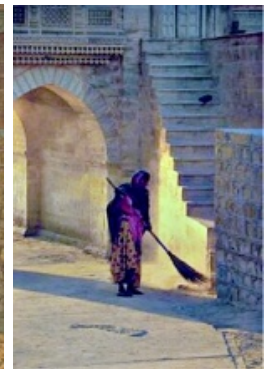

(d)

Fig. 6. This figure compares our method with existing methods. (a) The original image, (b) our greedy-based method $(\delta=2)$, (c) curvelet transformation [11, (d) method based on multi-scale retinex theory [9. Note that (c) leads to a noisy image while (d) changes the hue of the image significantly

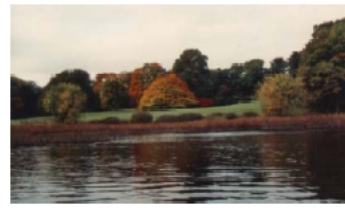

(a)

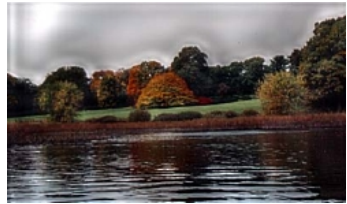

(d)

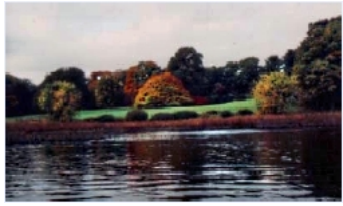

(b)

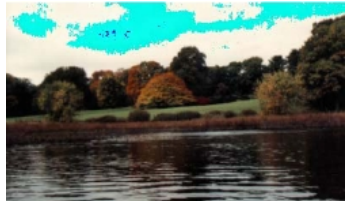

(e)

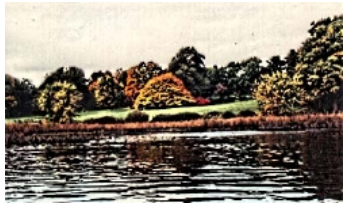

(c)

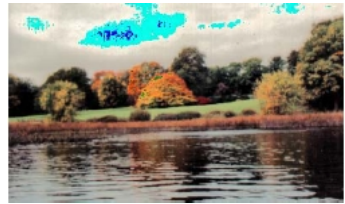

(f)

Fig. 7. (a) The original image, (b) our method, (c) multi-scale morphology method [5] - note the saturation artifacts that gives the image an unrealistic look, (d) Toet's method of multi-scale non-linear pyramid recombination 3 - note the halo artifacts at regions of large change in gradients, (e) global contrast stretching, (f) global histogram equalization - both (e) and (f) suffer from saturation artifacts and color blotches. 


\section{Results}

We show the effect of our algorithm on gray images, with varying values of the input parameter $\delta$ (Figure 2). We also show the difference between directly applying the algorithm to the three color channels (Figure 4) and our adaptation in Section 3 to ensure color gamut constraints (Figure [5). Figures 6 and 7 compare our method with other local and global contrast enhancement methods.

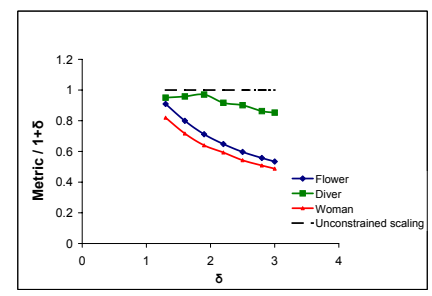

Fig. 8. Plot of $\frac{\alpha}{1+\delta}$ vs. $1+\delta$. The former is the average local contrast achieved while the latter is the average local contrast that would be achieved by scaling the image by $1+\delta$ without respecting constraints

Note that the objective function defined in Equation 1 or Equation 6 can also be used as an estimate of the average local contrast of an image, and hence, to evaluate the degree of enhancement achieved. According to the function, the maximum average local contrast that can be achieved without any constraints is given by $1+\delta$. However, imposing the constraints leads to a more practical average contrast value $\alpha<(1+\delta)$; as $\delta$ increases, the gap between $1+\delta$ and $\alpha$ widens since more pixels reach saturation and thus cannot achieve values close to $1+\delta$. Figure 8 plots the ratio $\frac{\alpha}{1+\delta}$ with $1+\delta$.

\section{Conclusion and Future Work}

We design a scalar objective function to describe the average local contrast of an image that has the potential to be used for estimating and evaluating the contrast of the image. We formulate the contrast enhancement problem as an optimization problem that tries to maximize the average local contrast of the image in a controlled fashion without saturation of colors. We present an efficient greedy algorithm controlled by a single input parameter $\delta$ to solve this optimization. Currently we are exploring adaptation of the same algorithm for tone mapping of high dynamic range images by changing $\delta$ spatially. Using the same concept, we can achieve seamless contrast enhancement of a selective region of interest in an image by varying the parameter $\delta$ smoothly over that region. Since our method works without explicitly segmenting the image either in the spatial or the frequency domain, it is very efficient. We are trying to exploit this efficiency to extend this method to video sequences, for which maintaining temporal continuity is of great importance. 


\section{References}

1. Oakley, J.P., Satherley, B.L.: Improving image quality in poor visibility conditions using a physical model for contrast degradation. IEEE Transactions on Image Processing 7 (1998) 167-179

2. Boccignone, G., Picariello, A.: Multiscale contrast enhancement of medical images. Proceedings of ICASSP (1997)

3. Toet, A.: Multi-scale color image enhancement. Pattern Recognition Letters 13 (1992) 167-174

4. Toet, A.: A hierarchical morphological image decomposition. Pattern Recognition Letters 11 (1990) 267-274

5. Mukhopadhyay, S., Chanda, B.: Hue preserving color image enhancement using multi-scale morphology. Indian Conference on Computer Vision, Graphics and Image Processing (2002)

6. Burt, P.J., Adelson, E.H.: A multiresolution spline with application to image mosaics. ACM Transactions on Graphics 2 (1983) 217-236

7. Hanmandlu, M., Jha, D., Sharma, R.: Localized contrast enhancement of color images using clustering. Proceedings of IEEE International Conference on Information Technology: Coding and Computing (ITCC) (2001)

8. Munteanu, C., Rosa, A.: Color image enhancement using evolutionary principles and the retinex theory of color constancy. Proceedings 2001 IEEE Signal Processing Society Workshop on Neural Networks for Signal Processing XI (2001) 393-402

9. Rahman, Z., Jobson, D.J., , Woodell, G.A.: Multi-scale retinex for color image enhancement. IEEE International Conference on Image Processing (1996)

10. Velde, K.V.: Multi-scale color image enhancement. Proceedings on International Conference on Image Processing 3 (1999) 584-587

11. Stark, J.L., Murtagh, F., Candes, E.J., Donoho, D.L.: Gray and color image contrast enhancement by curvelet transform. IEEE Transactions on Image Processing $12(2003)$

12. Hanmandlu, M., Jha, D., Sharma, R.: Color image enhancement by fuzzy intensification. Proceedings of International Conference on Pattern Recognition (2000)

13. Shyu, M., Leou, J.: A geneticle algorithm approach to color image enhancement. Pattern Recognition 31 (1998) 871-880

14. Fattal, R., Lischinski, D., Werman, M.: Gradient domain high dynamic range compression. ACM Transactions on Graphics, Proceedings of ACM Siggraph 21 (2002) 249-256

15. Prez, P., Gangnet, M., Blake, A.: Poisson image editing. ACM Transactions on Graphics, Proceedings of ACM Siggraph 22 (2003) 313-318

16. Valois, R.L.D., Valois, K.K.D.: Spatial Vision. Oxford University Press (1990)

17. Giorgianni, E.J., Madden, T.E.: Digital Color Management : Encoding Solutions. Addison Wesley (1998) 